\title{
A Driving Strategy Model of Self-Driving Cars
}

\author{
Haoge Liu*, Zeyu Shen, Wei Shang \\ School of Science, China University of Geosciences (Beijing), Beijing, China \\ Email:*690594105@qq.com
}

How to cite this paper: Liu, H.G., Shen, Z.Y. and Shang, W. (2017) A Driving Strategy Model of Self-Driving Cars. Journal of Computer and Communications, $\mathbf{5}$, $42-49$.

https://doi.org/10.4236/jcc.2017.55004

Received: February 8, 2017

Accepted: March 20, 2017

Published: March 23, 2017

Copyright $\odot 2017$ by authors and Scientific Research Publishing Inc. This work is licensed under the Creative Commons Attribution International License (CC BY 4.0).

http://creativecommons.org/licenses/by/4.0/

(c) (i) Open Access

\begin{abstract}
The self-driving cars are highly developed and about to meet the market, but the driving strategies and corresponding behaviors with others still need to be tested. In this paper, based on its characteristics and behaviors of manualdriving vehicles, we propose the driving strategies of manual-driving cars as well as self-driving cars. And we use the cellular automaton to simulate the traffic reality under different conditions, and to evaluate the efficiency of a road when self-driving cars are put into use. This research can be a reference by traffic planning and vehicles performance test, and further research can be designed in a model which can calculate the efficiency of a road when the percentage of self-driving cars are different.
\end{abstract}

\section{Keywords}

Self-Driving Cars, Driving Strategy, Cellular Automaton, Analog Simulation, Traffic Jam

\section{Introduction}

Self-driving, cooperating cars have been developed for years and already been tested on the road in some cities. Compared with manual-driving cars, they are equipped with automatic control system, combine them with high-resolution maps of the surrounding and status of others, they can always guide themselves to drive in optimal path.

A great benefit of self-driving, cooperating cars, of course, is to relieve the congestion of traffic. Because the volume of traffic exceeds the designed capacity of the road networks, numerous of drivers in large cities experience long delays during peak traffic hours. In some degree, self-driving, cooperating cars can increase capacity of highways without increasing number of lanes or roads.

However, since some governments yet haven't analyzed the address cooperation between self-driving cars, as well as the interaction between self-driving and non-self-driving vehicles, the real effects of these cars' behavior are still unmea- 
sured. Then it would be our job to formulate a feasible model to simulate operating condition of roads, and determine the solution to improve the efficiency when self-driving cars and manual-driving cars navigate cooperatively.

Urmson C's paper has showed that more than 1.2 million people die in traffic accidents annually [1]. The prevailing belief in the automotive industry is that the benefit of passive safety systems such as seat belts and air bags has reached a plateau. Hee Lee G presented a visual ego-motion estimation algorithm for selfdriving car equipped with a close-to-market multi-camera system, and their work leads to a novel 2-point minimal solution for the generalized essential matrix is obtained where the full relative motion including metric scale [2]. And Godthelp $\mathrm{H}$ has also involved the time-to-line-crossing (TLC) concept into driving strategy, which representing the time necessary for the vehicle to reach either edge of the driving lane, and it seems a valuable description [3].

Based on the pervious works, we need to solve following issues:

- Design a method to evaluate the efficiency of traffic flow, and use the cellular automaton to simulate the congestion degree of crowded road or clear road.

- Define optimal driving strategies for manual-driving cars and self-driving cars, and show the traffic reality for different cars.

\section{The Efficiency of Traffic Flow}

To measure the effects of traffic flow, we have to define a evaluate criterion for traffic flow itself. Generally speaking, drivers wish less cars on the road so they can speed up, or they can drive steadily in ideal velocity. But there always come unforeseen circumstances that cause a pause and every car around in the traffic stream has to slow their speed, this lead to a traffic jam, the circumstances can be showed as Figure 1 [4]. So we define a crowded road has a high density and low velocity of cars. On the contrary, in a clear road, either traffic density is low enough, or all cars move rapidly in a close team.

Traffic flow can be regarded as a particle flow consistent of vehicles, and it has three variables: quantity of flow $(Q)$, velocity $(V)$ and density of cars $(K)$. According to crowd flow equations [5], those variables have following relationship:

$$
Q=K * V
$$

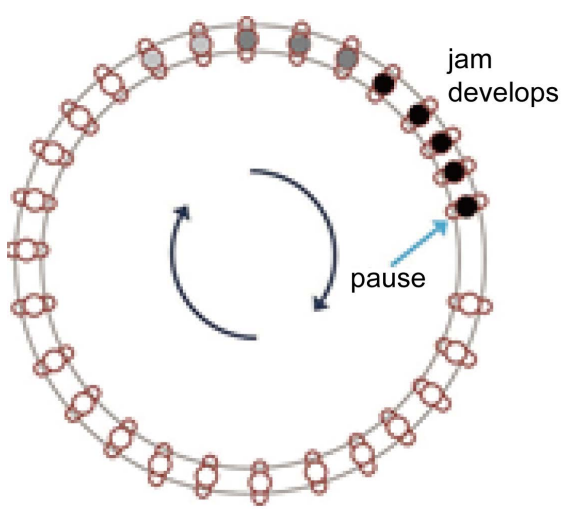

Figure 1. Traffic jam. 
To demonstrate better, we use $Q_{m}, K_{m}$, and $V_{m}$ to represent the value of corresponding variables when flow is maximized, and their relation can be exhibited by the Figure 2 .

As we can see in the equation and figure, the flow is positive correlation with $K$ and $V$, but there are inverse proportional relationship between velocity and density of cars, so the flow reach to $Q_{m}$ when $\mathrm{K}$ and $\mathrm{V}$ achieve a balanced state [6].

This phenomenon is easy to explain, drivers are informed to keep longer distance to each other when traffic speed is high, to prevent rear-end collision, then the density of roads will decrease. But self-driving, cooperating cars can keep move consistently with others, and keep high speed in high density, we will discuss its strategies in latter part. Now we can build a model to evaluate the efficiency of a road and effects on it.

When we evaluate the efficiency of traffic in a road, the conditions of peak time and daily average are not same. Based on the analysis above, we confirm two criteria to respectively evaluate the effects on the efficiency of traffic flow in daily average and peak time.
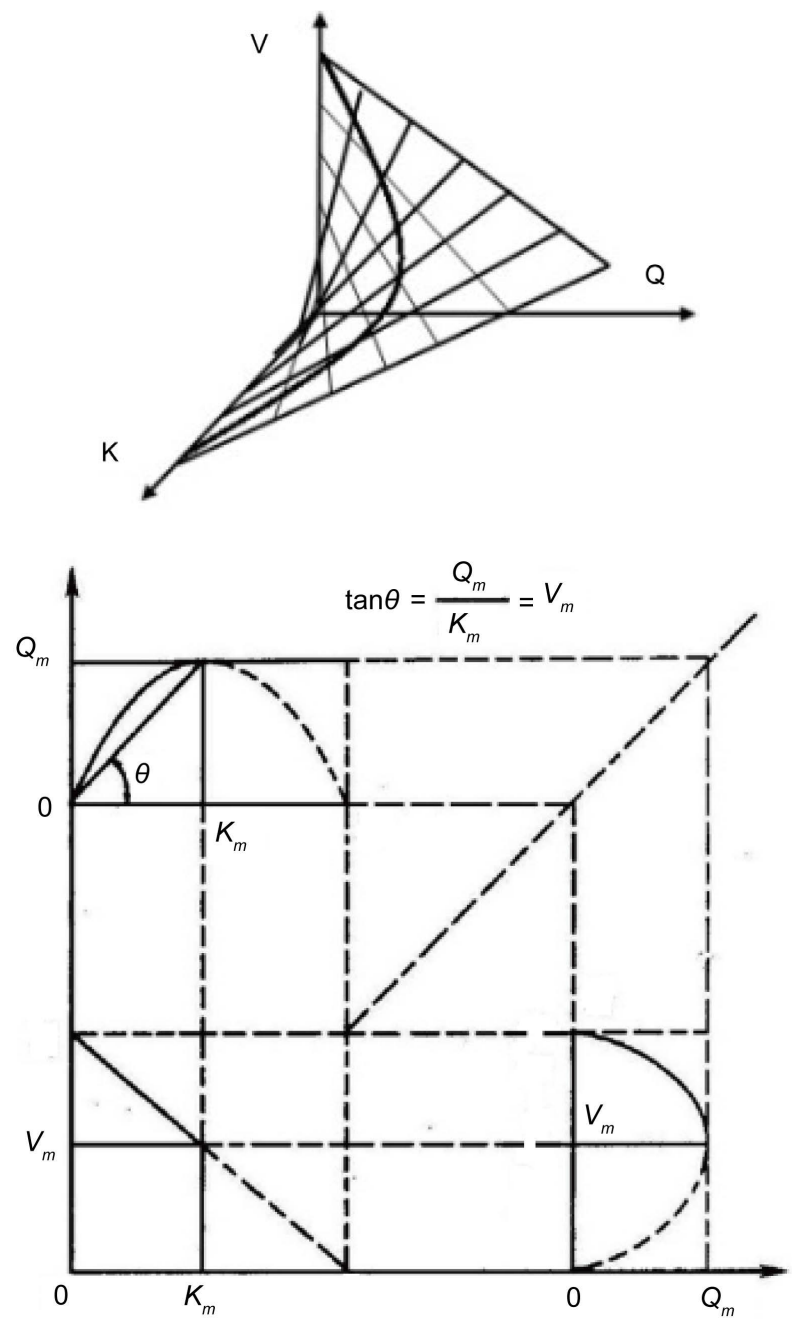

Figure 2. Relationship between flow, velocity and density. 
According to our assumption, people's route will be unchanged no matter which transportation they choose, and the number of vehicles will also be the same during to the same capacity of self-driving cars, so the average daily traffic volume of a road is constant or the flow is constant. In this condition, when density goes low, velocity goes high, efficiency of traffic will be improved. Therefore, a highly efficient road has a high-velocity and low-density traffic. We define density of cars $(K)$ as evaluation of traffic efficiency and EA as the effects on traffic in daily average:

$$
E A=\frac{K_{0}-K_{i}}{K_{0}} \%
$$

In this formula, $K_{0}$ represent the original density of roads in Washington, and it can be calculated by provided data. $K_{i}$ is the optimized density when we change the percentage of self-driving cars, and those data will be simulated in next part.

However, the state of traffic flow in peak time is inconsistent, in rush hours, every car tries to pass through the road in limited time. Even though the velocity of overall is improved, latter cars will keep pace immediately, and the whole density of flow will remain same. In this condition, if we want reduce the length of peak time, we should increase the number of passing cars in unit time, which means the velocity as well as the flow is increased, and efficiency of traffic will be improved. We define traffic flow $(Q)$ as evaluation of traffic efficiency and EP as the effects on traffic in daily average:

$$
E P=\frac{Q_{i}-Q_{0}}{Q_{0}} \%
$$

In this equation, $Q_{0}$ represent the original flow of roads in Washington, and it can be calculated by provided data. $Q_{i}$ is optimized flow and those data will be simulated by cellular automaton in next part.

Next we will define the strategies how self-driving and manual-driving vehicles move on highway.

\section{Driving Strategies of Vehicles}

\subsection{Strategies of Manual-Driving Vehicles}

$\mathrm{NaSch}$ model is a famous simple and useful model of cellular automaton target at traffic flow. It gives out a series of strategies how the position and speed change:

Step 1: Accelerate: $v=\min \left(v+1, v_{\max }\right)$, vehicles tend to accelerate when allowed to.

Step 2: Decelerate: $v=\min (v, d)$, slow down in case of accident, $d$ represents for the distance to the front car.

Step 3: Automatically decelerate: $v=\max (v-1,0)$ with a probability of $p d$, indicate to the result of road condition, the mental condition of the driver, and so on.

Step 4: Move: $x=x+v$, update the position of vehicles.

Here we made some changes: 
Change 1: Decelerate: $v=\min \left(v, \frac{d}{2}\right)$, the former computing method will cause accident when the front car is closer than $d$, and perform sudden brakes. In our model $d>d_{0}-\frac{d_{0}}{2}-\frac{d_{0}}{2^{2}}-\ldots-\frac{d_{0}}{2^{n}}-\ldots>d_{0}-\lim _{n \rightarrow \infty}\left(\frac{\frac{1}{2}-\frac{1}{2^{n}}}{1-\frac{1}{2}}\right)=0$, that means the vehicles will gradually and safely slow down.

Change 2: When calculate the distance to the front car, we use the position of the front car after this second instead of the current one, which prevents unnecessary decelerate.

Then we added changing-lane strategies to form a complete multi-lane model:

Step 1: If speed is limited by the front car, check if it's possible to move left; if possible, move left, else go to step 2 .

Step 2: Check if it's possible to move right; if possible, move right, else go to step 3.

Step 3: Slow down as stated in the single-lane model.

Step 4: If the front vehicle is far, accelerate.

Step 5: Automatically decelerate as stated in the single-lane model.

Now the model of manual-drive has complete. To simplify, we use automatically decelerate probability to account for all the reason why vehicles slow down, except for the influence of other vehicles. Therefore we don't need to consider the crossing, entrance and exit of highways.

\subsection{Strategies of Self-Driving Vehicles}

Firstly we assume that all of the strategies of manual-driving vehicles are also suitable for self-driving vehicles, which is to say that their basic behavior is similar. Meanwhile they also have several differences:

1) Self-driving vehicles have shorter reaction time, and their speed won't be influenced by mental factors. They can choose the best lane to keep fast. Therefore they have lower $p d$.

2) Self-driving vehicles can judge the traffic condition not only by vision, but also by data on the internet. So they can cooperate with each other to prevent congestion.

3) Self-driving cars give priority to dedicated lane (if provided), where they can keep fast and safe. All of actions on that lane are controlled by computer, not interrupted by manual-driving vehicles.

\section{Traffic Reality by Cellular Automaton}

Equip with the measure rules and driving strategies above, we can simulate the traffic reality based on real data in highway traffic, in this paper, we use the highway's data of Washington states, and we will use cellular automaton to simulate their traffic reailty.

A cellular automaton is a discrete model studied in computability theory, 
mathematics, physics, complexity science, theoretical biology and microstructure modeling. In the model of cellular automaton, we consider the status of the system in discrete time and space. For example, when we apply cellular automaton to traffic flow, we just consider the change in position, speed and other status of each vehicle in each second. We can get the traffic condition in every second, just like Figure 3 [7].

When simulating the traffic flow, we just choose a section of highway, which is just several miles in length. That is to say that the condition of road, vehicles and drivers and other influencing factors are consistent on this section of highway. Therefore the variables in this model are the maximum speed limit, the speed of the vehicles when they enter the section, the number of vehicles entering in one second, the number of lanes $(n I)$ and the strategies of the vehicles.

We can find the $Q_{0}$ of each section of highway in the given milepost table. To simplify the issue, we assume that whether in peak hours or average, the flow volume of both directions are the same. Therefore we can calculate the number of vehicles entering in one second $\left(V_{p s}\right)$ :

$$
V_{p s}= \begin{cases}\frac{Q}{2} * K * 3600 & \text { during peak hour }(K=0.08) \\ \frac{Q}{2} * \frac{1}{24} * 3600 & \text { average of a day }\end{cases}
$$

To simulate the influence of $V_{p s}$, we define $P_{i n}$ as the probability for each lane in each second if one vehicle enters, so $P_{i n}$ can be calculated as:

$$
P_{\text {in }}=\frac{V_{p s}}{n l} \cdot 100 \%
$$

Then let's consider the control strategies of $P_{i n}$ :

- To simulate the average status of a day, we believe that $Q_{i}$ won't be influenced by the condition of traffic flow (such as $v_{i}$ and $K_{i}$ ), so $P_{i n}$ is constant.

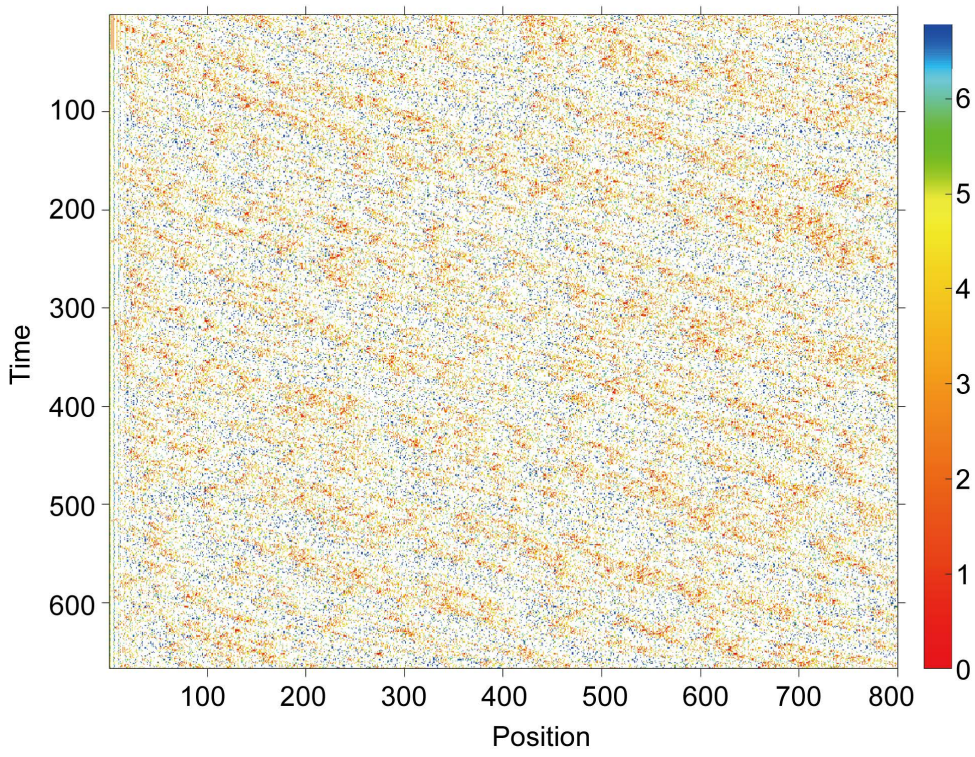

Figure 3. Vehicle speeds change over time, during peak hour (437 v/mile). Color represents speed, and red points are the slowest. 


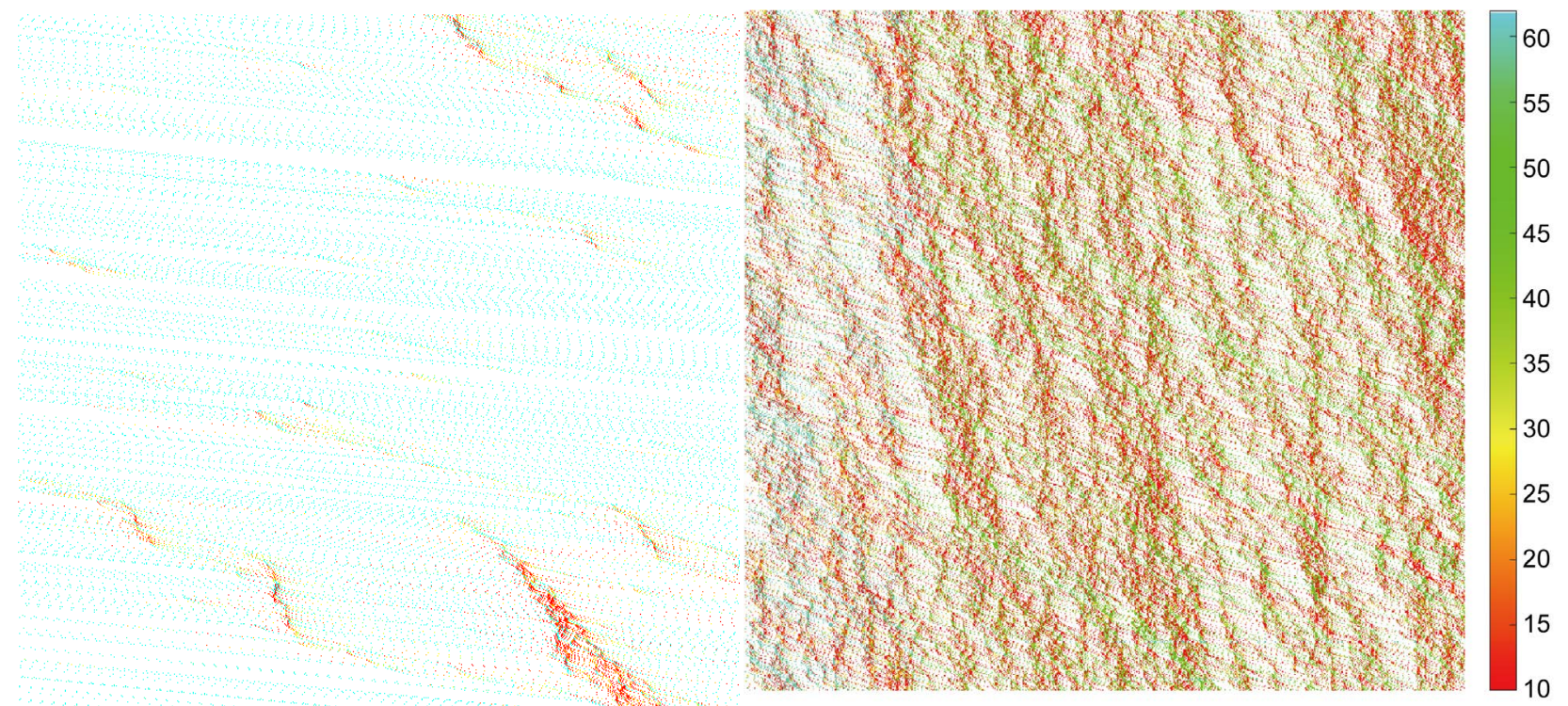

Figure 4. Traffic flow over time. Color represents speed. Left: Self-driving traffic flow, Right: Manual-driving traffic flow.

- To simulate the status of peak hour, we believe that all of people want to pass as quick as possible, in which condition the $P_{i n}$ will increase a lot, and $K_{i}$ also increases. We must control the density of road, so that the vehicles won't be too slow and the highway won't be too crowded. Thus $P_{i n}$ should decrease when $K_{i}$ is high, and oppositely increase.

Guided by strategies above and simulated by cellular automaton, we can show the traffic conditions for manual-driving cars and self-driving cars separately, and results are showed in Figure 4:

\section{Conclusion}

We manage to propose a practical method to simulate the traffic reality of a road, which is based on the driving strategy model of self-driving cars. By our method, we can evaluate the traffic efficiency for a road when the self-driving cars are put into use. And we use the cellular automaton to simulate the traffic reality under different conditions, and to evaluate the efficiency of a road when self-driving cars are put into use. This research can be a reference by traffic planning and vehicles performance test. Our future work, would be improving an optimal model that can simulate diffident percentage of self-driving cars of one road, and evaluate road's efficiency.

\section{References}

[1] Urmson, C. and Whittaker, W. (2008) Self-Driving Cars and the Urban Challenge. Intelligent Systems IEEE, 23, 66-68. https://doi.org/10.1109/MIS.2008.34

[2] Hee Lee, G., Faundorfer, F. and Pollefeys, M. (2013) Motion Estimation for Self-Driving Cars with a Generalized Camera. Proceedings of the IEEE Conference on Computer Vision and Pattern Recognition, Portland, 23-28 June 2013, 27462753. https://doi.org/10.1109/cvpr.2013.354

[3] Godthelp, H., Milgram, P. and Blaauw, G.J. (1984) The Development of a Time- 
Related Measure to Describe Driving Strategy. Human Factors, 26, 257-268.

[4] Kowszun, J. (2013) Jamitons: Phantom Traffic Jams. School Science Review, 95, 5361.

[5] Fang, Z, Lo, S.M. and Lu, J.A. (2003) On the Relationship between Crowd Density and Movement Velocity. Fire Safety Journal, 38, 271-283.

[6] National Research Council (US) (2010) HCM 2010: Highway Capacity Manual. 5th Edition, Transportation Research Board, Washington, DC.

[7] Kai, N., Wolf, D.E., Wagner, P., et al. (1997) Two-Lane Traffic Rules for Cellular Automata: A Systematic Approach. Physical Review E Statistical Physics Plasmas Fluids \& Related Interdisciplinary Topics, 58, 1425-1437.

Submit or recommend next manuscript to SCIRP and we will provide best service for you:

Accepting pre-submission inquiries through Email, Facebook, LinkedIn, Twitter, etc. A wide selection of journals (inclusive of 9 subjects, more than 200 journals)

Providing 24-hour high-quality service

User-friendly online submission system

Fair and swift peer-review system

Efficient typesetting and proofreading procedure

Display of the result of downloads and visits, as well as the number of cited articles Maximum dissemination of your research work

Submit your manuscript at: http://papersubmission.scirp.org/

Or contact jcc@scirp.org 